\title{
Bemerkungen zur Kalkflora des Kreises Ober dem Manhartsberge in Niederösterreich.
}

\author{
Von Hermann Kalbruner.
}

Obschon die Verhreitung von Kalligestein im K. 0 M. B. eine unlergeordnete ist, so zeigt sich doch an manchen Lokalitäten der Einfluss der Kalkunterlage auf die Vegetation. Am dentlichsten tritt dieser Einfluss hei jenem Konglomerat hervor, welches aus Geschieben von Alpenkalk und Wiener Sandstein, verbunden mit einem Kalkcement besteht.

Diese Felsart kömmt im südlichen Theile des Kreises vor, und erstreckt sich in geringer Ausdehnung unter dem Namen Göblinger Gebirge von ler Gobelsburgerhaide uber Gedersdorf und Rohrendorf his Gneixendort. Als kalkholde Pflanzen erscheinen dort Aronia rotundifolia, Linosyris vulgaris und besonders häulig Teucrium montanum. Dem Göblinger Gebirge gegenüber liegend am rechten Ufer der Donau nimmt dieses Kalkglomerat eine grössere Ausdehnung ein, bildet den Schiffberg, auch Wetterkreuz genannt, bei Hollenburg; auf welchem eine bedeutende Anzahl von Ialkpflanzen vorkommt, als: Equisetum Telmateja, Quercus Cerris, Daphne Cneorum, Teucrium montanum, Helianthemum oelandicum, Polygala Chamaebuxus, Cotoneaster tomentosa, Aronia rotundifolia.

In zweiter Linie ist in dieser Bezichung der körnige Kalkstein in Betracht zu ziehen, welcher Einlagerungen in krystallinische Schiefergebirge bildet. Eine Reihe von mehr oder weniger zu Tage tretenden Lagern von körnigem Kalkstein durchzieht in schiefer Richtung von Norden nach Süden den ganzen Kreis, beginnt bei Drosendorf und endigt bei Spitz an der Donau.

Dieser Urkalk ist eine grobkörnige krystallinische, langsam verwitternde Masse von weisser oder blaugrauer Farbe. In der geologischen Reichsanstalt in Wien wurde weisser und auch weissgrauer Kalkstein von Spitz untersucht, dessen Analyse ergab:

$$
\begin{aligned}
& 24 \text { pCt. in Salzsäure unlöslich } \\
& 73 \Rightarrow \text { liohlensauren Kalk } \\
& 2 \% \text { Thonerde und Eisenoxydul } \\
& \text { mehst Spuren von Billererde. }
\end{aligned}
$$

Auf besagtem Kallisteine finden sich bei Spitz Leontodon incanus, Helianlhemum oelandicum, Jurinea mollis, bei Aggsbach Teucrium montanum; und da die genannten Pflanzen auf Löss, Schiefer und Granitunterlage dieses Kreises noch nicht aufgefunden wurden, so ist die Arinalme zulassig, dass dieses Gestein das Vorkommen kalklicbender Pflanzen begiinstige.

Auf meinen zahlreichen botanischen Ausflügen im Waldviertel habe ich auf Kïnigem Falkstein nur bei Brunn in Walde Aspleniun viride und um Grossmotten Pyrola uniflora als solche Pflanzen angetroffen, wolche liallibold zu nennen sind. 
Eine Lokalität, wo der körnige Kalk in ausgedehnten Lagern auftritt, ist die Umgebung des Marktes Kottes; um zu erfahren, ob dort Repräsentanten der Kalkflora auftreten, unternahm ich im Monate Juni dieses Jahres eine botanische Exkursion in die dortige Gegend, deren Ergebniss ich mir im Nachstehenden mitzutheilen erlaube.

Bergrath Cžižek in seinen Erläuterungen zur geologischen Karte der Umgebungen von Krems 1853 schreibt über die Verwendbarkeit des körnigen Kalksteines: "Es ist schade, dass dieses reiche Material für schöne Bauwerke und andere Arbeiten ganz unbenützt liegen bleibt."

Seit dem Inslebentreten der grossartigen Bauten in Wien hat sich jedoch der Verbrauch von Werksteinen aus körnigem Kalkstein, hier und da unrichtig Granit genannt, sehr gesteigert; in einigen, von der Donau nicht zu weit entfernten Orten, wie bei Mühldorf, und besonders in Thale des kleinen Kremsflusses bei Kottes und Mahrbach bestehen mehrere Steinbrüche, in welchem Gegenstände zu Bauzwecken im Grossen erzeugt werden.

Ich untersuchte die Umgegend dieser Steinbrüche genau, konnte aber durchaus keine Pflanze auffinden, die als Kalkzeiger gelten könne; ja selbst direkte auf dem Schutte und im Gerölle dieses Gesteins entwickelt sich eine Vegetation, die durchaus nichts Eigenthümliches zeigt; es wachsen dort z. B. Leontodon hastilis Koch, Helianthemum vulgare Gärtn., Ajuga genevensis, Cerastium arvense, Senecio vulgaris, Rumex crispus und dergleichen mehr indifferente Pflanzen.

Ueber die Vegetationsverhältnisse der Umgegend von Kottes erlaube ich mir noch nachstehende Bemerkungen: Das westliche Waldviertel gehört bekanntlich zu den kälteren Theilen des Landes, daher auch um Kottes die Planzen ziemlich spät zur Entwicklung gelangen; der Roggen blühte heuer den 17 . Juni, also um drei Wochen später, als im Landbezirke; in Gürten standen zur selben Zeit Paeonia peregrina Mill. und Diclytra spectabilis $\mathrm{Dec}$. in voller Blüthe. Letzteres Gewächs wird in den Gürten des Waldviertels gerne kultivirt und es ist erwähnenswerth, wie diese vor wenigen Jahren noch seltene Zierpflanze sich so schnell hier eingebürgert hat.

Was den Feldhau betriff, so ist hier wie überall im Waldviertel die Dreifelderwirthschaft gebräuchlich, Roggen, Hafer und Brache bilden den Turnus. Die allgemeino Futterpflanze ist Trifolium pratense.

Des rauhen Klimas wegen fehlen hier feinere Obstsorten; Prunus insititia ist ein verbreiteter Baum, aus dessen Früchten Branntwein erzeugt wird. Die Wälder enthalten meistens Fichten mit Tannen und Föhren, auch kommen kleine Bestände von Birken und Buchen vor.

Der Fleiss der Landwirthe hat in neuerer Zeit die Menge der Ackerunkräuter bedeutend vermindert, von den die gewöhnlichen Raphanus Raphanistrum, Anthemis arvensis, Rhinanthus Christa galli, Spergula arvensis und Fedia dentata Vahl. sind.

Die im Thale gelegenen trockenen Hügel und Wiesen beherbergen milde nahrhafte Futterkräuter, besonders häufig findet man dort: 
Tragopogon orientalis, Helianthemum vulgare Gärtn., Polygonum Bistorta, Campanula patula, Crepis succisaefolia Tausch $\alpha_{.}$glabra. Die hochgelegene sogenannte Kottinger Haide enthält Sumpfwiesen, auf denen vorkommen: Eriophorum angustifolium, Carex ampullacea Good., C. panicea, C. stellulata Good., C. Oederi Retz, C. canescens, Gymnadenia conopsea R. Br., Orchis latifolia, a. majalis, Pinguicula vulgaris, Pedicularis sylvatica, Arnica montana, Thesium pratense Ehrh., Alchemilla vulgaris, Veratrum album.

An den Ufern der Kleinen Krems finden sich: Ranunculus aconitifolius, Chaerophyllum hirsutum, Geum rivale.

Die Flora der Wälder enthält: Cineraria rivularis W. Kit., Rosa alpina, Aquilegia vulgaris, Sanicula europaea, Soldanella montana Willd.

Demzufolge finden sich in der Umgebung von Kottes trotz des in so bedeutender Ausdehnung vorhandenen körnigen Kalksteines keine Pflanzen, welche als besonders kalkhold zu bezeichnen wären.

An landschaftlichen Reizen ist diese Gegend durchaus nicht arm, von Mahrbach zieht sich längs der Kleinen Krems bis Kottes ein lieblicher Thalweg; besondere Erwähnung verdient die herrliche Aussicht, die sich dem Beschauer von dem eine Viertelstunde von Kottes entfernten Steinbruche von Bernhardt darbietet; im Vordergrunde das friedliche Rundsthal mit dem Dörfchen Oetzbach, während sich im Hintergrunde der Jauerling mit seinem Flachrücken in ganzer Ausdehnung repräsentirt.

Langenlois, im Juli 1873.

\title{
Die Vegetations-Verhältnisse des mittleren und östlichen Ungarns und angrenzenden Siebenbürgens.
}

\author{
Von A. Kerner.
}

LXIII.

1189. Verbascum Thapsus L. - In Waldlichtungen und Holzschlägen. Im mittelungar. Berglande in der Matra bei Parád und Diosgyör; in der Pilisgruppe zwischen Visegrad und Sct. Andrae, bei $P$. Csaba und $P$. Szántó und auf dem Johannisberg bei Ofen. Im Bihariageb. am Wege zur Fasanerie bei Grosswardein, bei Vaskóh und Rézbánya. - Trachyt, Schiefer, Kalk, tert. Sandboden. 200-750 Met. - Im Tieflande von mir nicht beobachtet. Auch Kit. in Addit. 137 sagt von $V$. Thapsus L., in planiore Hungaria nuspiam vidi, nec in montosa frequens." Kitaibel's frühere Angaben in dem Itinerar der Marmar. Reise p. 40, wonach dieses Verbascum auf der Debrecziner Landhöhe und in der Tiefebene vorkommen soll, beziehen sich daher 